\title{
Hibernating myocardium: morphological correlates of inotropic stimulation and glucose uptake
}

\author{
D Pagano, J N Townend, D V Parums, R S Bonser, P G Camici
}

\begin{abstract}
Background-In patients with postischaemic left ventricular dysfunction, segments recovering function after revascularisation (hibernating myocardium) may not respond during dobutamine echocardiography, despite preserved $\left[{ }^{18} \mathrm{~F}\right] 2$-fluoro-2-deoxy-D-glucose (FDG) uptake at positron emission tomography.

Objective-To investigate whether this lack of response might reflect the degree of ultrastructural change in hibernating myocardium.

Methods-Transmural biopsies were obtained from 22 dysfunctional segments in 22 patients during coronary artery bypass grafting and examined by light and electron microscopy. Wall motion scores and coronary vasodilator reserve were assessed before and after coronary artery bypass grafting (CABG).

Results-Mean (SD) wall motion score improved in all segments following CABG (from 2.24 (0.4) to $1.55(0.4) ; \mathrm{p}<0.0001)$, confirming hibernating myocardium. In these segments myocardial blood flow (positron emission tomography with $\mathrm{H}_{2}^{15} \mathrm{O}$ ) before CABG was similar to that in normal volunteers $(1.02(0.24) v 1.02(0.23) \mathrm{ml} / \mathrm{min} / \mathrm{g})$, while the coronary vasodilator reserve was blunted (1.26 (0.7) v 3.2 (1.6); $\mathrm{p}<0.0001)$. Myocardial blood flow was unchanged after CABG, whereas coronary vasodilator reserve increased to $2.10(0.90)(p<0.0007)$. In hibernating myocardium myofibrillar loss, interstitial fibrosis, and glycogen-rich myocytes were more marked than in control donor hearts. On the basis of the response to dobutamine before CABG, two functional groups were identified: group A, segments with inotropic reserve $(n=15)$; group $\mathrm{B}$, segments without inotropic reserve $(\mathrm{n}=7)$. FDG uptake was similar in group A and group $\mathrm{B}$ $(0.40(0.1) v 0.44(0.1) \mu \mathrm{mol} / \mathrm{min} / \mathrm{g})$. In group B there was more myofibrillar loss $(26(8) \% v 11$ $(5) \% ; p=0.0009)$ and glycogen-rich myocytes $(28(11) \% v 17(10) \% ; p=0.02)$, whereas interstitial fibrosis, myocardial blood flow, and coronary vasodilator reserve were similar in the two groups. Myofibrillar loss was the only independent predictor of inotropic reserve $(p=0.01)$.

Conclusions-Hibernating myocardium is characterised by a reduced coronary vasodilator reserve which improves on revascularisation and shows a spectrum of ultrastructural changes that influence the response to dobutamine, while FDG uptake is invariably preserved.
\end{abstract}

(Heart 2000;83:456-461)

Cardiothoracic Surgical Unit, Queen Elizabeth Hospital, Birmingham, UK

D Pagano

R S Bonser

University

Department of

Cardiovascular

Medicine, Queen

Elizabeth Hospital

$\mathrm{J} \mathrm{N}$ Townend

Department of

Histopathology,

Papworth Hospital,

Cambridge, UK

D V Parums

MRC Cyclotron Unit, Imperial College School of Medicine, Hammersmith Hospital, Du Cane Road, London W14 0NN, UK P G Camici

Correspondence to: Professor Camici email: paolo.camici@ csc.mrc.ac.uk

Accepted 1 November 1999

Keywords: coronary artery disease; heart failure; myocardial viability; myocardial blood flow; positron emission tomography

In patients with coronary artery disease, chronically dysfunctional left ventricular myocardium which recovers contractile function after revascularisation has been defined as "hibernating." 2 Hibernating myocardium shows preserved exogenous glucose uptake and inotropic contractile reserve upon $\beta_{1}$ adrenergic stimulation. These properties are probed in clinical practice with positron emission tomography (PET) using the glucose analogue $\left[{ }^{18} \mathrm{~F}\right]$ 2-fluoro-2-deoxy-D-glucose (FDG), and by dobutamine echocardiography.

The morphological correlates of hibernating myocardium in man have been described as consisting of myocardial cells which are transformed from a functional state (rich in contractile material) to a "surviving" state (rich in glycogen material with loss of contractile proteins), ${ }^{3-6}$ with increased expression of glucose transporters. ${ }^{7}$ The loss of contractile material and the increase in interstitial fibrosis in hibernating myocardium may explain the lack of contractile response on $\beta_{1}$ adrenergic stimulation which has been reported in some cases. ${ }^{8-10}$

In addition, myocardial perfusion reserve is reduced in hibernating myocardium, ${ }^{11}$ and the severity of this impairment and its duration may influence the degree of ultrastructural adaptations and the response to $\beta_{1}$ adrenergic stimulation. ${ }^{92-14}$ Under these circumstances even a small increase in oxygen requirement, such as during low dose dobutamine infusion, cannot be met by an adequate increase in blood flow, so there is resultant ischaemia and an inability to increase contractile function. ${ }^{15} 16$

This study was therefore undertaken to investigate the relation between the inotropic response to dobutamine, myocardial perfusion, FDG uptake, and myocardial ultrastructure in patients with postischaemic heart failure and hibernating myocardium.

\section{Methods}

STUDY POPULATION

Patients

The patient population consisted of 22 subjects (three women, 19 men; mean (SD) age 57 (7) years) with multivessel coronary artery disease and chronic heart failure (New York Heart Association functional class $\geqslant$ III). All were in sinus rhythm and had suffered at least one Q wave myocardial infarct (range 1 to 3 ) at a 
Table 1 Patient characteristics

\begin{tabular}{lllllll}
\hline Patient & Biopsy & $\begin{array}{l}\text { WMSI } \\
\text { baseline }\end{array}$ & WMSI-dob & ICR & $\begin{array}{l}\text { WMSI } \\
\text { follow up }\end{array}$ & MGU \\
\hline 1 & Ant & 2.3 & 2.3 & 0 & 1.7 & 0.61 \\
2 & Ant $^{\star}$ & 1.3 & 1.3 & 0 & 1.0 & 0.26 \\
3 & Ant & 1.7 & 1.7 & 0 & 1.3 & 0.32 \\
4 & Ant & 2.3 & 2.3 & 0 & 2.0 & 0.58 \\
5 & Ant $^{\star}$ & 2.0 & 2.0 & 0 & 1.3 & 0.39 \\
6 & Ant $^{\star}$ & 2.3 & 2.3 & 0 & 2.0 & 0.43 \\
7 & Ant & 3.0 & 3.0 & 0 & 2.7 & 0.50 \\
8 & Ant & 2.7 & 1.3 & 1.4 & 1.3 & 0.29 \\
9 & Ant & 2.7 & 2.0 & 0.7 & 1.7 & 0.45 \\
10 & Ant & 1.7 & 1.0 & 0.7 & 1.3 & 0.26 \\
11 & Ant $^{\star}$ & 2.3 & 2.0 & 0.3 & 1.7 & 0.42 \\
12 & Ant & 2.0 & 1.7 & 0.3 & 1.3 & 0.41 \\
13 & Ant & 2.3 & 2.0 & 0.3 & 1.0 & 0.47 \\
14 & Lat $^{\star}$ & 2.7 & 2.0 & 0.7 & 1.3 & 0.52 \\
15 & Ant $^{\star}$ & 2.0 & 1.7 & 0.3 & 1.7 & 0.37 \\
16 & Ant $^{\star}$ & 2.0 & 1.3 & 0.7 & 1.0 & 0.45 \\
17 & Lat $^{\star}$ & 2.3 & 2.0 & 0.3 & 1.7 & 0.51 \\
18 & Ant $^{\star}$ & 2.7 & 2.3 & 0.4 & 2.0 & 0.53 \\
19 & Ant $^{\star}$ & 2.7 & 2.3 & 0.4 & 2.0 & 0.41 \\
20 & Ant $^{\star}$ & 3.0 & 2.0 & 1.0 & 2.0 & 0.25 \\
21 & Ant $^{\star}$ & 1.7 & 1.3 & 0.4 & 1.0 & 0.31 \\
22 & Ant $^{\star}$ & 2.0 & 1.7 & 0.3 & 1.3 & 0.40 \\
\hline
\end{tabular}

$\star$ Indicates presence of $\mathrm{Q}$ wave on ECG.

Ant, anterior wall; ICR, inotropic contractile reserve (see methods); Lat, lateral wall; MGU, myocardial glucose uptake $(\mu \mathrm{mol} / \mathrm{min} / \mathrm{g})$; WMSI, wall motion score index from the biopsied region (see methods for calculation); WMSI-dob, maximum response to dobutamine stimulation

mean of 30 (14) months (range 12 to 60) before the study. Patients were receiving treatment for heart failure, including angiotensin converting enzyme inhibitors and diuretics, but not $\beta$ blockers. The ejection fraction was 24 (7) $\%$ (range $10 \%$ to $37 \%$ ).

Normal control subjects

A group of 21 age and sex matched normal volunteers (mean age 53 (12) years) served as controls for the myocardial blood flow and coronary vasodilator reserve measurements. None of the controls had a history of cardiac disease, and all had a low risk profile, normal physical examination, normal resting ECG, and negative exercise tests at high workload.

Control biopsies

Transmural left ventricular biopsies were obtained from seven donors (aged 37 (8) years) during explantation for transplantation, before induction of ischaemic arrest.

STUDY PROTOCOL

Radionuclide ventriculography

Left ventricular ejection fraction was calculated at baseline and six months following coronary artery bypass grafting (CABG) by radionuclide ventriculography, using standard techniques. ${ }^{17}$

Transthoracic and dobutamine stress echocardiography

Baseline and dobutamine studies (5 and 10 $\mu \mathrm{g} / \mathrm{kg} / \mathrm{min}$ ) were performed as previously described ${ }^{18}$ by an experienced echocardiographer blinded to all the clinical details of the patients. Resting wall motion was reassessed six months following CABG.

The left ventricle was divided in 16 segments and a four point scoring system was used to analyse wall motion. ${ }^{19}$ The left ventricle was then divided into four regions: anterior (basal, mid, and apical segments), septum (basal, mid, apical, mid-anterior, and basal anterior septal segments), lateral (basal, mid, and apical segments), and inferoposterior (basal, mid, and apical inferior and basal and mid posterior segments). The wall motion score index for each region (WMSI) was calculated as the average of the scores of the segments contained in each region. ${ }^{19}$ The maximum inotropic contractile reserve with dobutamine (dWMSI) was calculated as the difference between WMSI at baseline (WM-basal) minus WMSI with dobutamine (WM-dob), defined as the maximum improvement in WMSI obtained with either dose of dobutamine.

In a random subset of 10 patients the interobserver and the intraobserver $\kappa$ agreement was assessed (interobserver $\kappa=0.82$; intraobserver $\kappa=0.90)$. A wall was considered hibernating if an improvement in WMSI was demonstrated at the follow up study.

PET measurement of myocardial blood flow, coronary vasodilator reserve, and myocardial glucose uptake

The PET studies were performed before revascularisation within one week of the dobutamine echocardiography study using an ECAT 931-08/12 scanner (CTI Inc, Knoxville, Tennessee, USA) as previously described. ${ }^{20}$ Myocardial blood flow was measured using oxygen-15 labelled water $\left(\mathrm{H}_{2}{ }^{15} \mathrm{O}\right) .^{20}{ }^{21} \mathrm{To}$ account for the prevailing haemodynamic conditions, baseline myocardial blood flow was corrected for the rate-pressure product as previously reported. ${ }^{20}$ In 19 of 22 patients myocardial blood flow was also measured two minutes after the intravenous administration of dipyridamole $(0.56 \mathrm{mg} / \mathrm{kg}$ over four minutes $){ }^{22}$ Coronary vasodilator reserve was calculated as dipyridamole/baseline myocardial blood flow. In these patients a repeat measurement of myocardial blood flow and coronary vasodilator reserve was made six months after CABG. Myocardial glucose uptake was measured during hyperinsulinaemic euglycaemic clamp, using the glucose analogue FDG. ${ }^{20}$ PET images were reconstructed and analysed as previously described ${ }^{20}$ to match sectors corresponding to the echo images.

MYOCARDIAL BIOPSY SAMPLING

Transmural left ventricular biopsies were obtained in all 22 patients during coronary surgery using a Tru-Cut biopsy needle (14 gauge) from the dysfunctional area supplied by a stenotic coronary artery ( $>75 \%$ stenosis) that was amenable to revascularisation. In 20 patients the biopsies were taken from the anterior and in two from the lateral left ventricular wall. In each case, two contiguous specimens were taken away from any macroscopic scar tissue before induction of ischaemic arrest. All these 22 dysfunctional regions improved in function after CABG - that is, they were truly hibernating (table 1). Control transmural biopsies were obtained from the left ventricular apex of seven donors during explantation for transplantation, before the induction of ischaemic arrest. 
TISSUE PREPARATION AND ANALYSIS

Light microscopy

One biopsy was placed immediately in $10 \%$ formalin at room temperature (neutral buffered formol/saline) for 18-24 hours to avoid contraction band artefact, and routinely processed in a commercial processor (Tissue-Tek, Bayer Diagnostics, Newbury, Berkshire, UK). The specimen was dehydrated in $50 \%, 70 \%$, $95 \%$, and $100 \%$ ethanol, cleared in xylene, and embedded in molten paraffin wax $\left(\sim 60^{\circ} \mathrm{C}\right)$ in a $3 \times 4 \mathrm{~cm}$ plastic cassette. Paraffin sectioning was done at $5 \mu \mathrm{m}$ for light microscopy.

Tissue morphology was assessed after haematoxylin and eosin (H\&E) staining. Elastic van Gieson (EVG) staining was used to assess the degree of fibrosis and elastin destruction within tissue sections. Periodic acid Schiff (PAS) before and after diastase digestion was used to assess intracellular lipofuscin and glycogen.

Light microscopic analysis of the centre of the biopsies was undertaken to exclude edge fixation artefact, counting only cells in which the nucleus was present in the plane of section, and using conventional planimetry. The percentage area of fibrosis was determined using a grid method, as previously described. ${ }^{3423}$ Briefly, a special grid was used with vertical and horizontal lines providing 117 intersections. According to the principles of morphometry, counting of the number of intersections overlying a certain structure results in a quantitative determination of the surface of the structure under investigation in relation to the surface of the entire tissue under the square grid. The total number of intersections was regarded as $100 \%$, and the intersections counted in the connective tissue were expressed as the percentage of the entire tissue within the limits of the grid. Blood vessels and neural tissue were disregarded. Cells in which more than $10 \%$ of the sarcomeres were replaced by glycogen (PAS) were classified as glycogen rich. ${ }^{24}$

\section{Electron microscopy}

Small pieces of tissue $(2 \times 4 \times 2 \mathrm{~mm})$ were fixed in $4 \%$ glutaraldehyde in 0.1 sodium cacodylate and $0.05 \% \mathrm{CaCl}_{2}$. Tissue was then rinsed three times in $0.1 \mathrm{M}$ sodium cacodylate buffer for 10 minutes each time and then post-fixed in $1 \%$ osmium tetroxide in cacodylate buffer for one hour. After this, it was rinsed in buffer for one to two hours, dehydrated in graded ethanols, rinsed in propylene oxide for 30 minutes, and embedded in epoxy resin containing the following: $2 \mathrm{ml}$ Polybed 812; $2 \mathrm{ml}$ Araldide $506 ; 5 \mathrm{ml} \mathrm{DDSA}$, and $0.18 \mathrm{ml}$ DMP-30 (CIBA, Duxford, Cambridgeshire, UK). Tissue was incubated in a 1:1 mixture of plastic-propylene oxide for 12 hours and then in plastic for two hours before placing it in a $60^{\circ} \mathrm{C}$ oven for 48 hours. Sections $1 \mu \mathrm{m}$ thick were analysed at magnifications between 3300 and 23000 . Morphometric analysis was performed as described by other groups ${ }^{23}{ }^{25}$ on the electronmicrographs. A magnification of 23000 was used for volume fractions and myofibrillar loss, defined as the percentage of cells showing a greater than $10 \%$ reduction in myofibrils.

The analysis was performed by an experienced cardiac pathologist (DVP) blinded to the nature of the biopsy (patient or control), clinical details, and the results of other investigations.

The study was approved by the local ethics committee, and written informed consent obtained from all patients before the study. The radiation exposure was licensed by the UK Administration of Radioactive Substances Advisory Committee (ARSAC). The investigations conform with the principles outlined in the Declaration of Helsinki.

\section{STATISTICAL ANALYSIS}

Continuous data are expressed as mean (SD) or as median and interquartile range (IQR), as appropriate. Continuous data were tested for Gaussian distribution with the Shapiro-Wilk test $^{26}$ and subsequently compared using the unpaired $t$ test (Gaussian) or the MannWhitney U test (non-Gaussian). Myocardial perfusion data were compared with the Kruskal-Wallis test. The univariate association between response to dobutamine (response or no response) and the potential predictors was assessed with the unpaired $t$ test or MannWhitney $\mathrm{U}$ test, as appropriate, and with univariate logistic regression. Stepwise logistic regression was then performed including predictors with $\mathrm{p}$ values not greater than 0.05 in the univariate analysis, and keeping predictors with $\mathrm{p}$ values not greater than 0.05 in the multivariate model. Changes before and after CABG were compared using the paired $t$ test. A value $<0.05$ (two sided) was considered statistically significant. Simple linear regression analysis with Pearson's correlation coefficient was performed to investigate the relation between two continuous variables.

\section{Results}

\section{FUNCTIONAL OUTCOME}

Six months after CABG the regional WMSI in the areas where the myocardial biopsy was obtained improved in all cases (from 2.24 (0.40) to $1.55(0.40) ; \mathrm{p}<0.0001)$, thus confirming the presence of hibernating myocardium (table 1). The global left ventricular ejection fraction also improved, from 24 (7)\% to $33(8) \%(\mathrm{p}<0.0001)$.

\section{MYOCARDIAL BLOOD FLOW}

Control subjects

Myocardial blood flow was homogeneously distributed throughout the ventricle. Baseline myocardial blood flow was $1.02(0.23) \mathrm{ml} /$ $\mathrm{min} / \mathrm{g}$ and increased to 3.1 (1.3) $\mathrm{ml} / \mathrm{min} / \mathrm{g}$ $(\mathrm{p}<0.0001)$ after dipyridamole. Coronary vasodilator reserve was 3.2 (1.6).

\section{Patients}

In the hibernating myocardium, baseline myocardial blood flow was comparable to that in the control subjects $(1.02(0.24) \mathrm{ml} / \mathrm{min} / \mathrm{g})$, whereas the response to dipyridamole was significantly blunted $(1.26 \quad(0.6) \mathrm{ml} / \mathrm{min} / \mathrm{g}$, $\mathrm{p}<0.0001 v$ controls). Coronary vasodilator 


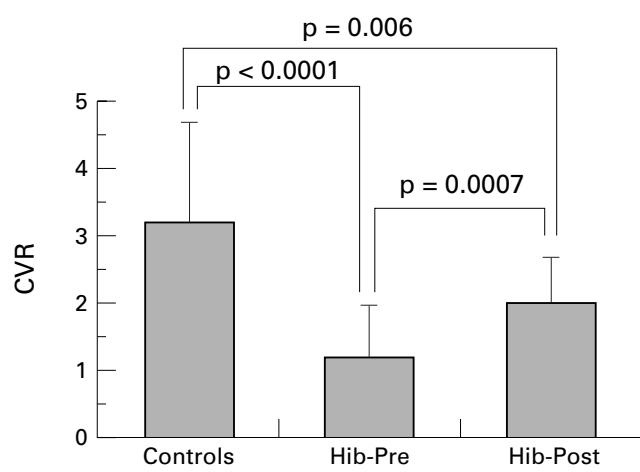

Figure 1 Bar chart showing the coronary vasodilator reserve (CVR) in normal volunteers (controls) and in hibernating myocardium before (Hib-Pre) and six months after coronary artery bypass grafting (Hib-Post).

reserve was also markedly reduced compared with controls (1.26 (0.7), $\mathrm{p}<0.0001$, fig 1). Six months after CABG, baseline myocardial blood flow was unchanged $(1.07(0.2) \mathrm{ml} / \mathrm{min} /$ $\mathrm{g})$, whereas the response to dipyridamole $(2.1$ (0.9) $\mathrm{ml} / \mathrm{min} / \mathrm{g} ; \mathrm{p}=0.004 v$ pre-CABG) and coronary vasodilator reserve (2.0 (0.8); $\mathrm{p}=0.0007 v$ pre-CABG) were significantly increased (fig 1).

\section{MORPHOLOGICAL CHANGES IN HIBERNATING} MYOCARDIUM

Compared with myocardium from the donor hearts (controls), biopsies obtained from the hibernating regions showed morphological changes; these included interstitial fibrosis, loss of myofibrillar content and myofibrillar disarray, accumulation of cytosolic glycogen, and depletion of sarcoplasmic reticulum. In the hibernating regions the volume fraction of myofibrillar loss was $14.5 \%$ (IQR 9 to 25 ), the percentage of glycogen-rich cells was $21 \%$ (IQR 11 to 32), and the percentage of interstitial fibrosis was $11 \%$ (IQR 6 to 19). In the control hearts the volume fraction of myofibrillar

Table 2 Quantitative morphological changes in group $A$ (response to dobutamine) and $B$ (no response)

\begin{tabular}{|c|c|c|c|c|c|}
\hline & \multicolumn{2}{|l|}{ Group $A$} & \multicolumn{2}{|l|}{ Group B } & \multirow[b]{2}{*}{$p$ Value } \\
\hline & $\begin{array}{l}\text { Mean } \\
(S D)\end{array}$ & $\begin{array}{l}\text { Median } \\
(I Q R)\end{array}$ & $\begin{array}{l}\text { Mean } \\
(S D)\end{array}$ & $\begin{array}{l}\text { Median } \\
(I Q R)\end{array}$ & \\
\hline Damaged myofibrils (\%) & $11(5)$ & $13(7$ to 16$)$ & $26(8)$ & 28 (11 to 33$)$ & 0.0009 \\
\hline Glycogen-rich cells (\%) & $17(10)$ & $12(10$ to 26$)$ & $28(11)$ & $24(22$ to 42$)$ & 0.028 \\
\hline Interstitial fibrosis (\%) & $14(9)$ & $11(6$ to 21$)$ & $13(9)$ & 11 (5 to 18$)$ & 0.850 \\
\hline
\end{tabular}

IQR, interquartile range.

Table 3 Comparison between group $A$ (response to dobutamine) and $B$ (no response)

\begin{tabular}{llll}
\hline & Group $A$ & Group B & p Value \\
\hline LVEF (\%) & $24(7)$ & $24(8)$ & 0.90 \\
WMSI & $2.3(0.4)$ & $2.1(0.5)$ & 0.85 \\
MBF (ml/min/g) & $1.0(0.2)$ & $1.0(0.3)$ & 0.34 \\
CVR & $1.3(0.7)$ & $1.1(0.4)$ & 0.14 \\
FDG $(\mu \mathrm{mol} / \mathrm{min} / \mathrm{g})$ & $0.40(0.1)$ & $0.44(0.1)$ & 0.42 \\
Heart rate, basal (beats/min) & $86(8)$ & $86(10)$ & 0.90 \\
Heart rate, peak (beats/min) & $98(9)$ & $98(13)$ & 0.70 \\
RPP, basal & $10383(4300)$ & $9471(1353)$ & 0.14 \\
RPP, peak & $12140(2266)$ & $10990(1513)$ & 0.10
\end{tabular}

Values are mean (SD).

CVR, coronary vasodilator reserve in the biopsied region (corrected for rate-pressure product); FDG, $\left[{ }^{18} \mathrm{~F}\right]$-fluorodeoxyglucose uptake in the biopsied region; LVEF, global left ventricular ejection fraction; $\mathrm{MBF}$, myocardial blood flow in the biopsied region (corrected for rate-pressure product); RPP, rate-pressure product (basal and peak dobutamine); WMSI, wall motion score index in the biopsied region. loss was 3\% (IQR 2 to 6), glycogen-rich cells $4 \%$ (IQR 2 to 5), and interstitial fibrosis $1 \%$ (IQR 0 to 3) (all p $<0.0001 v$ hibernating myocardium).

GLUCOSE UPTAKE AND INOTROPIC CONTRACTILE RESERVE OF HIBERNATING MYOCARDIUM

The myocardial glucose uptake in chronically dysfunctional regions which recovered function after revascularisation (that is, hibernating myocardium) was in all cases greater than 0.25 $\mu \mathrm{mol} / \mathrm{min} / \mathrm{g}$ (that is, viable myocardium ${ }^{18}{ }^{27}$ ) and on average it was $0.41(0.11) \mu \mathrm{mol} / \mathrm{min} / \mathrm{g}$ (table 1). The response of hibernating myocardium to dobutamine before CABG identified two groups: those with inotropic contractile reserve $(\delta \mathrm{WMSI}=0.57(0.3)$, IQR 0.3 to 1.6 , $\mathrm{n}=15$, group $\mathrm{A})$, and those without inotropic contractile reserve ( $\delta \mathrm{WMSI}=0 ; \mathrm{n}=7$, group B). In the latter group there was increased myofibrillar loss and glycogen-rich myocytes, whereas the percentage of interstitial fibrosis did not differ between the two groups (table 2). The two groups had similar myocardial glucose uptake and were matched for age (57(9) and 57(4) years), heart rate, and rate-pressure product at baseline and peak dipyridamole infusion, baseline WMSI, and ejection fraction and myocardial blood flow results (table 3 ).

Stepwise logistic regression identified the volume fraction of myofibrillar loss as the only independent predictor of inotropic contractile reserve $(p=0.01)$, and a significant relation was identified between the degree of myofibrillar loss and inotropic contractile reserve in the hibernating myocardium $(R=-0.65$; $\mathrm{p}=0.001)$.

CORRELATION BETWEEN INOTROPIC CONTRACTILE RESERVE AND FUNCTIONAL OUTCOME

WMSI was lower six months after CABG in both groups (group A, from $2.3(0.4)$ to 1.5 (0.4), $\mathrm{p}<0.0001$; group $\mathrm{B}$, from $2.1(0.5)$ to $1.7(0.6) ; \mathrm{p}<0.001)$, but the improvement in WMSI was greater in group A $(0.8(0.4) v 0.4$ $(0.2), p<0.05)$ (fig 2). A relation between the WMSI during dobutamine and following CABG was, however, only found in group A $(R=0.68, \mathrm{p}=0.005)$.

\section{Discussion}

This study shows that, while the uptake of glucose (as measured by FDG-PET) was preserved in all hibernating segments, the inotropic contractile response to dobutamine was sometimes absent; it was influenced by the degree of myofibrillar loss and required the preservation of a relatively normal myocyte ultrastructure. These findings may offer an explanation for the false negative results of dobutamine stress echocardiography previously reported by $\mathrm{us}^{18}$ and in other studies of subjects with significant left ventricular dysfunction. ${ }^{9} 102829$ On the other hand, the preservation of basic myocyte functions involved in glucose uptake and phosphorylation, including changes in glucose transporter expression, ${ }^{7}$ allow exogenous glucose utilisation by the ischaemic myocyte, despite derangement of the contractile apparatus. ${ }^{30}{ }^{31} \mathrm{In}$ 

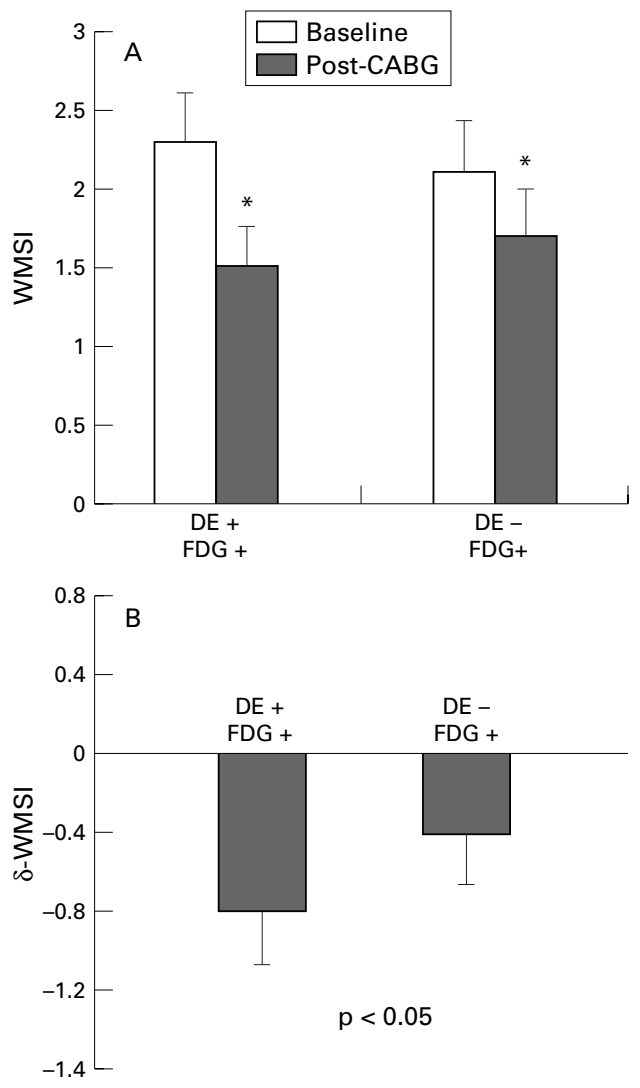

Figure 2 (A) Bar chart showing improvements in wall motion score index (WMSI) six months after coronary artery bypass grafting (CABG) in hibernating segments with inotropic contractile reserve $(D E+)$ and preserved FDG uptake (FDG+) and in those without inotropic contractile reserve (DE-) but preserved glucose uptake $(F D G+) .{ }^{*} p<0.001$ v baseline in each group. (B) Bar chart comparing the absolute improvements in WMSI in the two groups.

our patients with hibernating myocardium the degree of interstitial fibrosis, which was similar to that reported by others, ${ }^{5}$ did not influence the response to $\beta_{1}$ adrenergic stimulation. The morphological changes of hibernating myocardium have been described as progressive cellular structural dedifferentiation..$^{23}$ Our findings suggest that this could be associated with a stepwise "functional dedifferentiation." In "mild" myocardial hibernation, resting contraction is impaired but cellular metabolic function and the capacity to respond to $\beta_{1}$ adrenergic stimulation are preserved. A more severe form of hibernating myocardium is characterised by reduced basal contractile function with no response to $\beta_{1}$ adrenergic stimulation, but preserved glucose uptake, enhanced glycogen storage, and increased expression of glucose transporters. This is probably also reflected in different degrees of ultrastructural abnormalities as demonstrated by the results of the present study (table 2).

It has been proposed that the response to dobutamine stimulation of hibernating myocardium depends upon myocardial blood flow and coronary vasodilator reserve..$^{912-14} 1633$ However, myocardial blood flow and coronary vasodilator reserve were comparable in the myocardial regions of groups $\mathrm{A}$ and $\mathrm{B}$ and were not predictive of response to dobutamine. In accordance with previous observations, our study shows that baseline myocardial blood flow in hibernating myocardium is within normal limits, ${ }^{102034}$ whereas coronary vasodilator reserve is severely blunted. ${ }^{34}$ In addition, our data demonstrate that following coronary revascularisation baseline myocardial blood flow remains unchanged, whereas coronary vasodilator reserve is significantly improved. These findings confirm the hypothesis that continuing and sustained ischaemia is unlikely to be the trigger for hibernation. More probably, the myocardium is subjected to repeated episodes of ischaemia during everyday life as myocardial oxygen demand exceeds supply, with subsequent contractile dysfunction (stunning), culminating in myocardial hibernation and chronic ventricular dysfunction. ${ }^{35}$ It could be speculated that the transition from a "mild" to a "severe" form of hibernation does not depend on a different severity of coronary artery disease and coronary vasodilator reserve reduction, but may simply reflect different temporal stages of the condition. In addition, this might alter the expression of glucose transporters ${ }^{7}$ to enhance exogenous glucose uptake and maximise intracellular glycogen storage, as this could provide protection against the ischaemic insults, ${ }^{36}$ thus preserving myocyte viability.

The improvement in regional myocardial function six months after surgical revascularisation was greater in patients with preserved inotropic contractile reserve than in patients with viable myocardium but no inotropic response to dobutamine. In the latter group, however, revascularisation proved to be still worthwhile, as demonstrated by the significant improvements in global left ventricular function experienced by the patients. In addition, complete reversibility of contractile dysfunction may also depend upon the nature and the degree of morphological alterations of hibernating myocardium, as reported by other groups. ${ }^{6738}$ This may influence the time course of recovery of function, and a single postoperative study may underestimate the amount of hibernating myocardium.

\section{CLINICAL IMPLICATIONS}

Patients with heart failure, such as the population of our study, constitute the group in whom clinical decision to recommend coronary revascularisation, heart transplant, or continued medical treatment remains difficult. The presence of hibernating myocardium that is not "arousable" by $\beta_{1}$ adrenergic stimulation, but has preserved glucose uptake, may lead to rejection of some patients for revascularisation who may have more viability than can be detected by assessment of inotropic contractile reserve alone. As the severity of ultrastructural changes influences the degree of contractile function recovery after CABG it could be important to investigate the time course of such changes and the mechanisms underlying the transition from the "mild" to the more "severe" form of hibernating myocardium. This could have implications upon the timing for revascularisation. 
STUDY LIMITATIONS

An important limitation of this study, dictated by obvious safety reasons, was the size of the biopsies. As changes within the left ventricular walls may not be homogeneous, the sample might not be representative for the whole wall. To minimise this problem, we obtained biopsies from the most dysfunctional areas in an attempt to analyse the site with the most marked changes. We did not specifically examine the histopathological changes within the subendocardial and subepicardial layers; this could have provided further information regarding the transmural relevance of the ultrastructural changes detected.

The biopsy and fixation procedures are known to give rise to artefactual ultrastructural morphological changes, such as myofibril "contraction band" artefacts. In this study, morphological assessment of the biopsies was undertaken following immediate and optimal tissue fixation processing and sectioning. Analysis of the centre of the biopsy was undertaken away from the edges, which are prone to fixation artefacts. The morphological indices chosen were those that excluded these recognised artefacts, and the analysis was done with no knowledge of the identity of the biopsies and clinical details of the patients studied.

1 Diamond G, Forrester J, deLuz P, Wyatt H, Swan H. Postextrasystolic potentiation of ischaemic cardiomyopathy by atrial stimulation. Am Heart f 1978;95:204-9.

2 Rahimtoola SH. A perspective on the three large multicenter randomized clinical trials of coronary artery bypass surgery for chronic stable angina. Circulation 1985; 72(suppl V):V-123-35.

3 Borgers $M$, Thone F, Wouters L, et al. Structural correlates of regional myocardial dysfunction in patients with critical of regional myocardial dysfunction in patients with critical Pathol 1993;2:237-45.

4 Flameng W, Wouters L, Sergeant P, et al. Multivariate analysis of angiographic, histologic, electrocardiographic data in patients with coronary artery disease. Circulation 1984;70:7-17.

5 Depré C, Vanoverschelde J, Melin J, et al. Structural and metabolic correlates of the reversibility of chronic left ventricular ischemic dysfunction in humans. Am $\mathcal{f}$ Physio 1995;268:H1265-75.

6 Elsasser AM, Schlepper M, Klovekorn W-P, et al. Hibernating myocardium. An incomplete adaptation to ischemia. Circulation 1997;96:2920-31.

7 Brosius F, Nguyen N, Egert S, et al. Increased sarcolemmal glucose transporter abundance in myocardial ischaemia. Am F Cardiol 1997;80:77-84A.

8 Heusch G, Schulz R. Hibernating myocardium: a review. $f$ Mol Cell Cardiol 1996;28:2359-72.

9 Bonow R. Identification of viable myocardium. Circulation 1996;94:2674-8.

10 Gerber B, Vanovershelde J-L, Bol A, et al. Myocardial blood flow, glucose uptake, and recruitment of inotropic reserve in chronic left ventricular ischemic dysfunction. Implications for the pathophysiology of chronic myocardial hibernation. Circulation 1996;94:651-9.

11 Camici PG, Wijns W, Borgers M, et al. Pathophysiological mechanisms of chronic reversible left ventricular dysfunction due to coronary artery disease (hibernating myocardium). Circulation 1997;96:3205-14.

12 McGillem M, DeBoe S, Friedman H, et al. The effects of dopamine and dobutamine on regional function in the presence of rigid coronary stenoses and subcritical impairement of reactive hyperemia. Am Heart $\mathcal{F} 1988 ; 115$ : 970-7.

13 Marzullo P, Parodi O, Sambuceti G, et al. Residual coronary reserve identifies viability in patients with wall motion abnormalities. I Am Coll Cardiol 1995;26:342-50.
14 Picano E, Ostojic M, Varga A, et al. Combined low dose dypiridamole-dobutamine stress echocardiography to identify myocardial viability. Am f Cardiol 1996;27:1422-8.

15 Schulz R, Guth B, Pieper K, et al. Recruitment of an inotropic reserve in moderately ischemic myocardium at the expense of metabolic recovery. A model of short-term hibernation. Circ Res 1992;70:1282-95.

16 Chen C, Chen L, Prada J, et al. Incremental doses of dobutamine induce a biphasic response in dysfunctional left ventricular regions subtending coronary stenoses. left ventricular regions subte
Circulation 1995;92:756-66.

17 Zaret BL, Wackers FJ. Nuclear cardiology. N Engl f Med 1993;329:855-63.

18 Pagano D, Bonser R, Townend J, et al. Predictive value of dobutamine echocardiography and positron emission tomography in identifying hibernating myocardium in patients with postischaemic heart failure. Heart 1998;79:281-8.

19 Segar D, Brown S, Sawada S, et al. Dobutamine stress echocardiography: correlation with coronary lesion severity as determined by quantitative angiography. $7 \mathrm{Am}$ Coll Cardiol 1992;19:1197-202.

20 Marinho N, Keogh B, Costa D, et al. Pathophysiology of chronic left ventricular dysfunction. New insights from the measurement of absolute myocardial blood flow and glucose utilisation. Circulation 1996;93:737-44.

21 Araujo L, Lammertsma A, Rhodes C, et al. Non-invasive quantification of regional myocardial blood flow in normal volunteers and patients with coronary artery disease using oxygen-15 labelled carbon dioxide inhalation and positron emission tomography. Circulation 1991;83:875-85.

22 Uren N, Melin J, De Bruyne B, et al. Relation between myocardial blood flow and the severity of coronary artery stenosis. N Engl f Med 1994;330:1782-8.

23 Ausma J, Cleutjens J, Thoné F, et al. Chronic hibernating myocardium: interstitial changes. Mol Cell Biochem 1995; 147:35-42.

24 Maes A, Flameng W, Nuyts J, et al. Histological alterations in chronically hypoperfused myocardium. Correlation with PET findings. Circulation 1994;90:735-45.

$25 \mathrm{Li} \mathrm{Q}$, MacAuley M, Landymore R, et al. Morphometric analysis on myocardial injury related to the use of high volume potassium cardioplegic solution during ischaemic arrest. Pathol Res Pract 1992;188:668-71.

26 Shapiro S, Wilk M. An analysis of variance test for normality. Biometrika 1965;52:591.

27 Fath-Ordoubadi F, Beatt K, Spyrou N, et al. Efficacy of coronary angioplasty for the treatment of hibernating myocardium (in press)

28 Chan R, Lee K, Calafiore $\mathrm{P}$, et al. Comparison of dobutamine echocardiography and positron emission tomography in patients with chronic ischaemic left ventricular dysfunction. $\mathcal{f}$ Am Coll Cardiol 1996;27:1601-7.

29 Perrone-Filardi P, Pace L, Prastraro M, et al. Assessment of myocardial viability in patients with chronic coronary artery disease. Rest-4-hour-24-hour 201-thallium tomography versus dobutamine echocardiography. Circulation 1996;94:2712-19.

30 Camici PG, Ferranini E, Opie L. Myocardial metabolism in ischaemic heart disease: basic principles and applications to imaging by positron emission tomography. Progr Cardiovasc Dis 1989;32:217-38.

31 Depré C, Vanovershelde J-L, Taegtmeyer H. Glucose for the heart. Circulation 1999;99:578-88.

32 Ausma J, Furst D, Shivalkar B, et al. Molecular changes of titin in left ventricular dysfunction as a result of chronic hibernation. $\mathcal{f ~ M o l ~ C e l l ~ C a r d i o l ~ 1 9 9 5 ; 2 7 : 1 2 0 2 - 1 2 . ~}$

33 Beller A. Comparison of 201-thallium scintigraphy and low-dose dobutamine echocardiography for the noninvasive assessment of myocardial viability. Circulation 1996;94:2681-4.

34 Vanoverschelde J-L, Wijns W, Depré C, et al. Mechanism of chronic regional postischaemic dysfunction in humans. New insights from the study of noninfarcted collateraldependent myocardium. Circulation 1993;87:1513-23.

35 Camici PG, Rimoldi O. Myocardial hibernation vs repetitive stunning in patients. Cardiol Rev 1999;7:39-43.

36 McElroy D, Walker W, Taegtmeyer H. Glycogen loading mproves left ventricular function of the rabbit heart after hypothermic ischemic arrest. F Appl Cardiol 1989;4:45565 .

37 Schwarz E, Schoendube F, Kostin S, et al. Prolonged myocardial hibernation exacerbates cardiomyocyte degeneration and impairs recovery of function after revascularisation. F Am Coll Cardiol 1998;31:1018-26.

38 Shivalkar B, Maes A, Borgers M, et al. Only hibernating myocardium invariably shows early recovery after coronary revascularization. Circulation 1996;94:308-15. 